\title{
Mutation and haplotype analyses of the $M U T$ gene in Japanese patients with methylmalonic acidemia
}

\author{
Osamu Sakamoto $\cdot$ Toshihiro Ohura • \\ Yoichi Matsubara $\cdot$ Masaki Takayanagi . \\ Shigeru Tsuchiya
}

Received: 23 July 2006/ Accepted: 26 September 2006/ Published online: 31 October 2006

(C) The Japan Society of Human Genetics and Springer 2006

\begin{abstract}
Methylmalonic acidemia (MMA) is caused by a deficiency in the activity of L-methylmalonylCoA mutase (MCM), a vitamin B12 (or cobalamin, $\mathrm{Cbl}$ )-dependent enzyme. Apoenzyme-deficient MMA (mut MMA) results from mutations in the nuclear gene MUT. Most of the MUT mutations are thought to be private or restricted to only a few pedigrees. Our group elucidated the spectrum of mutations of Japanese mut MMA patients by performing mutation and haplotype analyses in 29 patients with mut MMA. A sequence analysis identified mutations in $95 \%(55 / 58)$ of the disease alleles. Five mutations were relatively frequent (p.E117X, c.385 + 5G > A, p.R369H, p.L494X, and p.R727X) and four were novel (p.M1V, c.753_753 + 5delGGTATA, c.1560G > C, and c.2098_2099delAT). Haplotype analysis suggested that all of the frequent mutations, with the exception of p.R369H, were spread by the founder effect. Among 46 Japanese patients investigated in the present and previous studies, $76 \%$ (70/92) of the mutations were located in exons $2,6,8$, and 13 . This finding - that a limited number of mutations account
\end{abstract}

O. Sakamoto $\cdot$ T. Ohura $(\bowtie) \cdot$ S. Tsuchiya

Department of Pediatrics,

Tohoku University School of Medicine,

1-1 Seiryo-machi, Aoba-ku,

Sendai 980-8574, Japan

e-mail: tohura@mail.tains.tohoku.ac.jp

Y. Matsubara

Department of Medical Genetics,

Tohoku University School of Medicine,

Sendai, Japan

M. Takayanagi

Chiba Children's Hospital, Chiba, Japan for most of the mutations in Japanese mut MMA patients - is in contrast with results of a previous study in Caucasian patients.

Keywords Methylmalonic academia .

L-Methylmalonyl-CoA mutase

\section{Introduction}

Methylmalonic acidemia (MMA) is an autosomalrecessive disorder of propionate metabolism caused by a defect in the isomerization of L-methylmalonyl-CoA to succinyl-CoA. The reaction is catalyzed by L-methylmalonyl-CoA mutase (MCM, EC 5.4.99.2), an enzyme which requires adenosylcobalamin (AdoCbl) as a cofactor (Fenton et al. 2001). MMA is classified into two forms: one resulting from a defect in the MCM apoenzyme (mut MMA or vitamin $\mathrm{B}_{12}$-unresponsive MMA; MIM 251000) and another resulting from a defect in the steps leading to AdoCbl synthesis ( $c b l$ MMA or vitamin $\mathrm{B}_{12}$-responsive MMA) (Rosenblatt and Fenton 2001). Typical MMA is characterized clinically by lethargy, vomiting, and hypertonia with abnormal movements, and biochemically by an accumulation of methylmalonic acid in the tissues and body fluid associated with hyperammonemia and ketoacidosis.

MCM is encoded by a single gene, $M U T$, which has been located to 6p21. MUT consists of 13 exons spanning $35 \mathrm{~kb}$ and it produces a $2.7-\mathrm{kb}$ mRNA. To date, more than 100 disease-causing mutations in the human MUT gene have been reported (Ledley and Rosenblatt 1997; Acquaviva et al. 2005; Martinez et al. 2005), most of which seem to be unique or restricted to 
only a few pedigrees. However, there have been reports of specific mutations among various ethic groups, including p.G717V in blacks (Adjalla et al. 1998), p.N219Y in Caucasians (Acquaviva et al. 2001), and p.R108C in Hispanics (Worgan et al. 2006). Ogasawara et al. (1994b) reported a relatively high incidence of p.E117X in Japanese patients and, more recently, Kobayashi et al. (2006) identified the plural occurrence of each of six mutations (p.L494X, p.R93H, p.E117X, p.R369H, p.G648D, and c.385 + 5G > A) in another Japanese population.

In study reported here, we performed mutation and haplotype analyses on 29 Japanese patients with mut MMA to examine the spectrum of mutations within the population and explore the possibility of a molecular diagnosis.

\section{Methods}

Patients

Twenty-nine apparently unrelated mut MMA patients were studied in the present investigation. There were no consanguineous marriages among the parents of these patients. Patient 6 was a Brazilian descended from Japan immigrants. All of the patients with available clinical information had been symptomatic during their neonatal or infantile periods. Responsiveness to vitamin B12 was not found in all of the patients except patient 7 . The oral administration of vitamin B12 in patient 7 reduced the urinary excretion of methylmalonic acid clinically, and the fibroblasts from this patient showed an increased incorporation of ${ }^{14} \mathrm{C}$-propionate - from 7 to $40 \%$
- following the administration of vitamin B12 in vitro (case 2 in Kakinuma et al. 1985). This patient was classified as $c b l$ MMA on the basis of these findings, but neither the MMAA nor $M M A B$ mutation was found (Yang et al. 2004). Patient 23 exhibited one of the mutations (p.V669E), but another mutation was not found in the previous study (cell No. 69 in Mikami et al. 1999). The diagnoses of MMA were confirmed by urinary organic acid analysis using gas chromatography and mass spectrometry. The MCM assay was performed in most cases by measuring the isomerization of L-methylmalonyl-CoA to succinylCoA by means of high-performance liquid chromatography (Kikuchi et al. 1989), and MCM activity was undetectable in all of those assayed. We could not successfully subclass the patients into $m u t^{0}$ or $m u t^{-}$by this method.

Direct sequencing of the $M U T$ genes

Genomic DNA was extracted from cultured fibroblasts, EBV-transformed lymphoblasts, or leukocytes with the aid of a Sepa Gene Kit (Sanko Junyaku, Tokyo, Japan). All coding exons, including franking introns in MUT, were amplified by PCR (Table 1). To facilitate the cycle sequencing analysis, the KS primer sequence and M13 reverse primer sequence were attached to the $5^{\prime}$ ends of the sense primers and antisense primers, respectively. The PCR products were directly sequenced using a Big Dye Primer Cycle Sequencing kit and an ABI 310 Genetic Analyzer (PE Applied Biosystems, Foster City, Calif.).

The Ethics Committee of the Tohoku University School of Medicine approved this study.

Table 1 Primers for the amplification of the MUT gene

\begin{tabular}{llll}
\hline Sense primer $^{\mathrm{a}}$ & & Antisense primer $^{\mathrm{b}}$ & \\
\hline MUT-Ex2-KS & KS-GAGTAGCTCCTATTTCCCAC & MUT-Ex2-Rev & Rev-GAGTGAATATCATCTTTACA \\
MUT-Ex3-KS & KS-ACCTTGATTCCAGACTCTTG & MUT-Ex3-Rev & Rev-CTACATTCAAGGAACTATAG \\
MUT-Ex4-KS & KS-AGTCCTGATGATGGTTCATG & MUT-Ex4-Rev & Rev-ATCTAAATCTAGCCTGACAT \\
MUT-Ex5-KS & KS-TGTACGTGCACTGATCTTAA & MUT-Ex5-Rev & Rev-CTTGTGCCACATTGCTCAGA \\
MUT-Ex6-KS & KS-GCTATTCTGAAGCTTAATAT & MUT-Ex6-Rev & Rev-TATAAATCTTGACTTGTAAG \\
MUT-Ex7-KS & KS-TGATGTTTATTTAATTCTGT & MUT-Ex7-Rev & Rev-GTGCATCCATGTATGTGAAA \\
MUT-Ex8-KS & KS-CTCAGATTGGGATTTGCTGA & MUT-Ex8-Rev & Rev-CACCTCATGCTGTTGTAAGG \\
MUT-Ex9-KS & KS-ATGCTATGCATCAGGGTCTA & MUT-Ex9-Rev & Rev-ACATGGTTTACAGGATCAAC \\
MUT-Ex10-KS & KS-GAATTGGATGCATAAAGGCA & MUT-Ex10-Rev & Rev-TTTCTCAGTTGTATGTAAGG \\
MUT-Ex11-KS & KS-CTTGAAAGATTTGCTGTGAA & MUT-Ex11-Rev & Rev-TACCAGTTACCAGGAGATGT \\
MUT-Ex12-KS & KS-GCCCATTAGTATGTTCTGAA & MUT-Ex12-Rev & Rev-ACACTGTCCACTTTTAGACC \\
MUT-Ex13-KS & KS-TGCCAGTAGTATACCAGTTG & MUT-Ex13-Rev & Rev-GAAGACATAGCTTTACTCTC \\
\hline
\end{tabular}

${ }^{\text {a }}$ KS, CGAGGTCGACGGTATCG

${ }^{b}$ Rev, CAGGAAACAGCTATGAC 


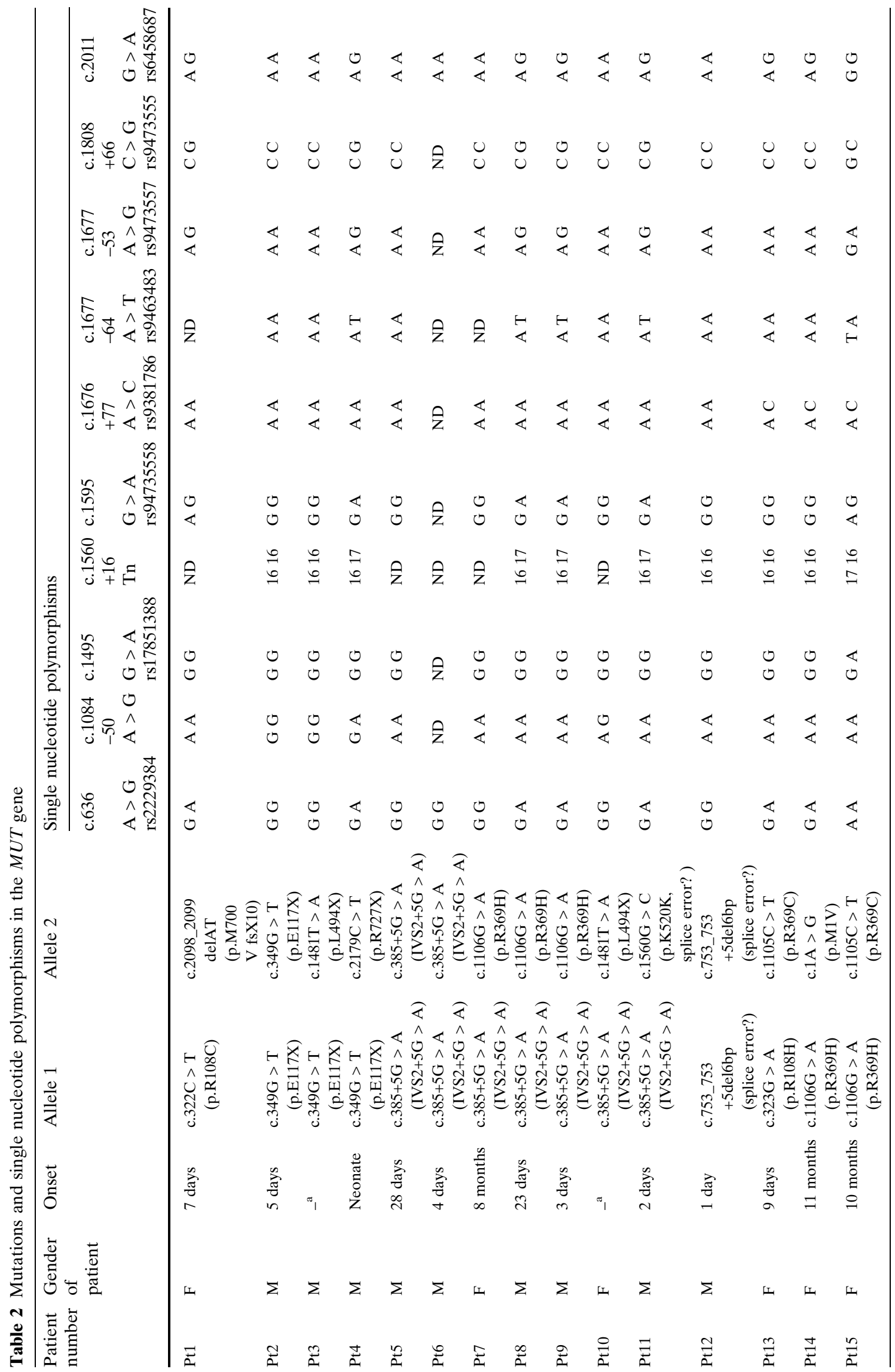




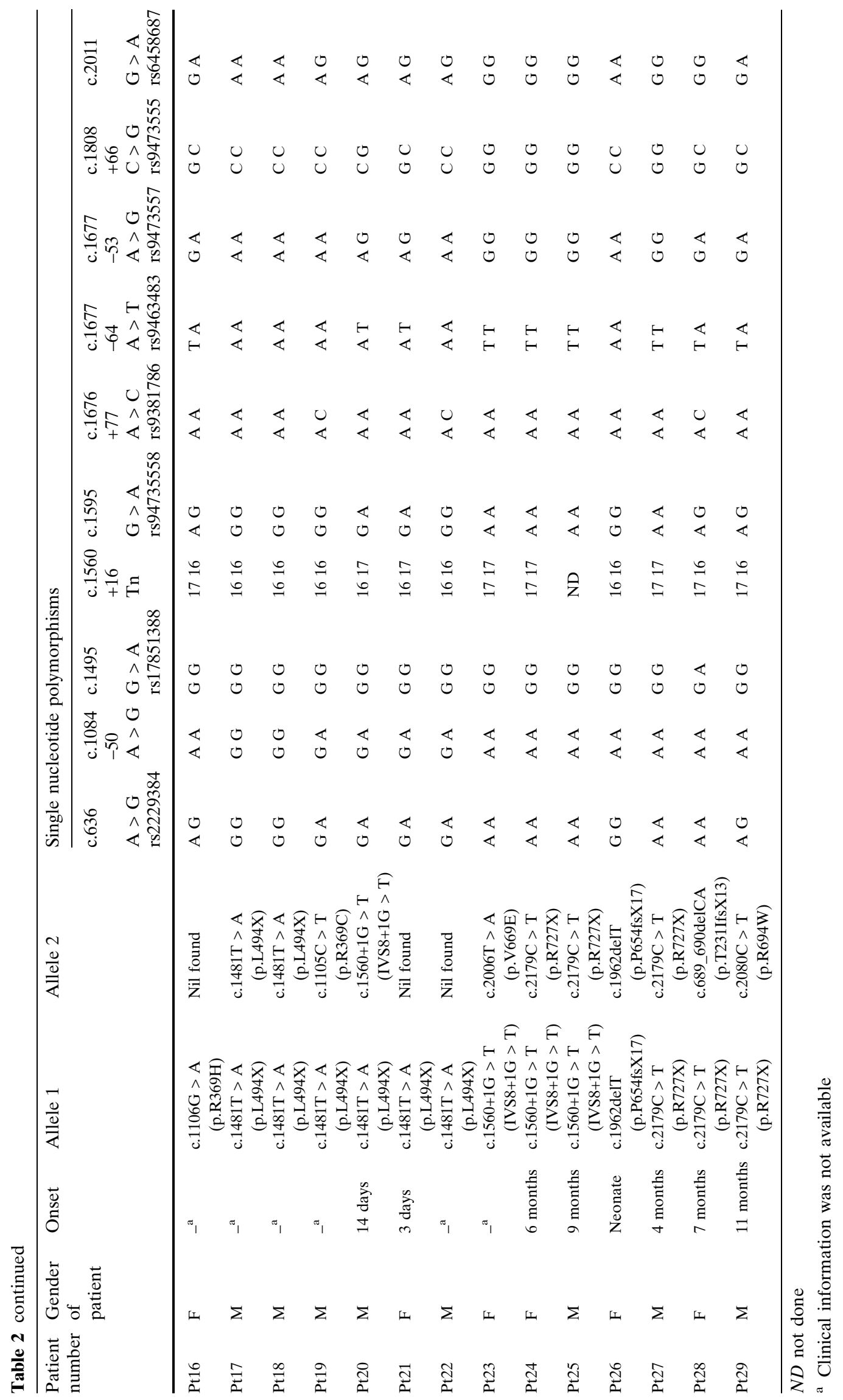




\section{Results and discussion}

Twenty-nine mut MMA patients were studied for mutation analysis. Sequence analysis identified mutations in $95 \%(55 / 58)$ of the disease alleles (Table 2), with 17 mutations being identified in total. Three of the patients $(16,21$, and 22) had only one mutation as a heterozygous change each.

Four mutations were novel (p.M1V, c.753_753 + 5delGGTATA, c.1560G > C, and c.2098_2099delAT). The mutation in the translation initiation codon, M1V, has been reported to be pathogenic in other diseases (Lyonnet et al. 1992; Cheadle et al. 1994). The presence of a splice donor site in intron 3 of c.753_753 + 5delGGTATA suggests that this deletion plays a pathogenic role. The sequence flanking this deletion exhibits an intrastrand complementarity (CAAAGGTATACTTTG). It is hypothesized that c.753_753 + 5delGGTATA is associated with the formation of the hairpin loop structure in a single-strand DNA (Robinson et al. 1997). A c.1560G > C substitution was identified at the $3^{\prime}$ end of exon 8 that appeared to result in missense-mediated splicing errors. A two-base deletion, c.2098_2099delAT, resulted in a frame-shift and a premature termination.

The total allelic frequency of four mutations (c.385 + 5G > A, p.R369H, p.L494X, and p.R727X) was $55 \%$ (32/58). The p.E117X mutation, previously reported as a relatively frequent mutation in Japanese, was found in four alleles (7\%) in this study. The mutations p.R93H and p.G648D each appeared more than once in the data from Kobayashi et al. (2006), whereas our data revealed neither. The mutations p.G717V (common mutation in black) and p.N219Y (common mutation in Caucasians) were not found, and p.R108C (common mutation in Hiapanic) was detected in only one allele in this study. Figure 1 summarizes the mutations found in 46 Japanese patients (Ogasawara et al. 1994a, b; Toyo-Oka et al. 1995; Mikami et al. 1999; Kobayashi et al. 2006). Worgen et al. (2006) identified exons 2, 3, 6, and 11 as mutation clusters, whereas our data on Japanese patients indicated that $76 \%(70 / 92)$ of the mutations were located in exons (and franking introns) 2, 6, 8, and 13.

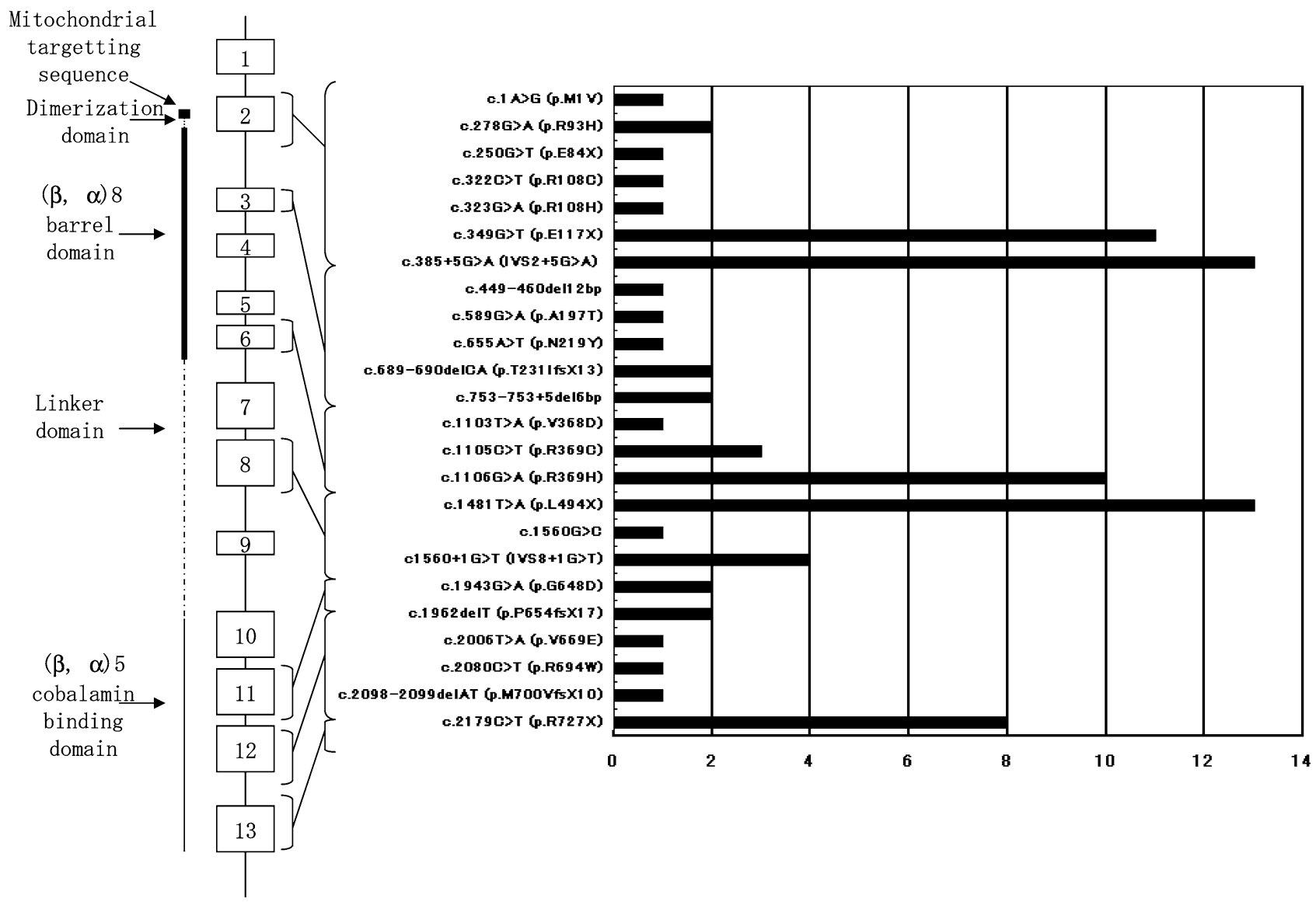

Fig. 1 The distribution of mutations found in 46 Japanese mut MMA patients (Ogasawara et al. 1994a, b; Toyo-Oka et al. 1995; Mikami et al. 1999; Kobayashi et al. 2006; and this study) 
J Hum Genet (2007) 52:48-55

53

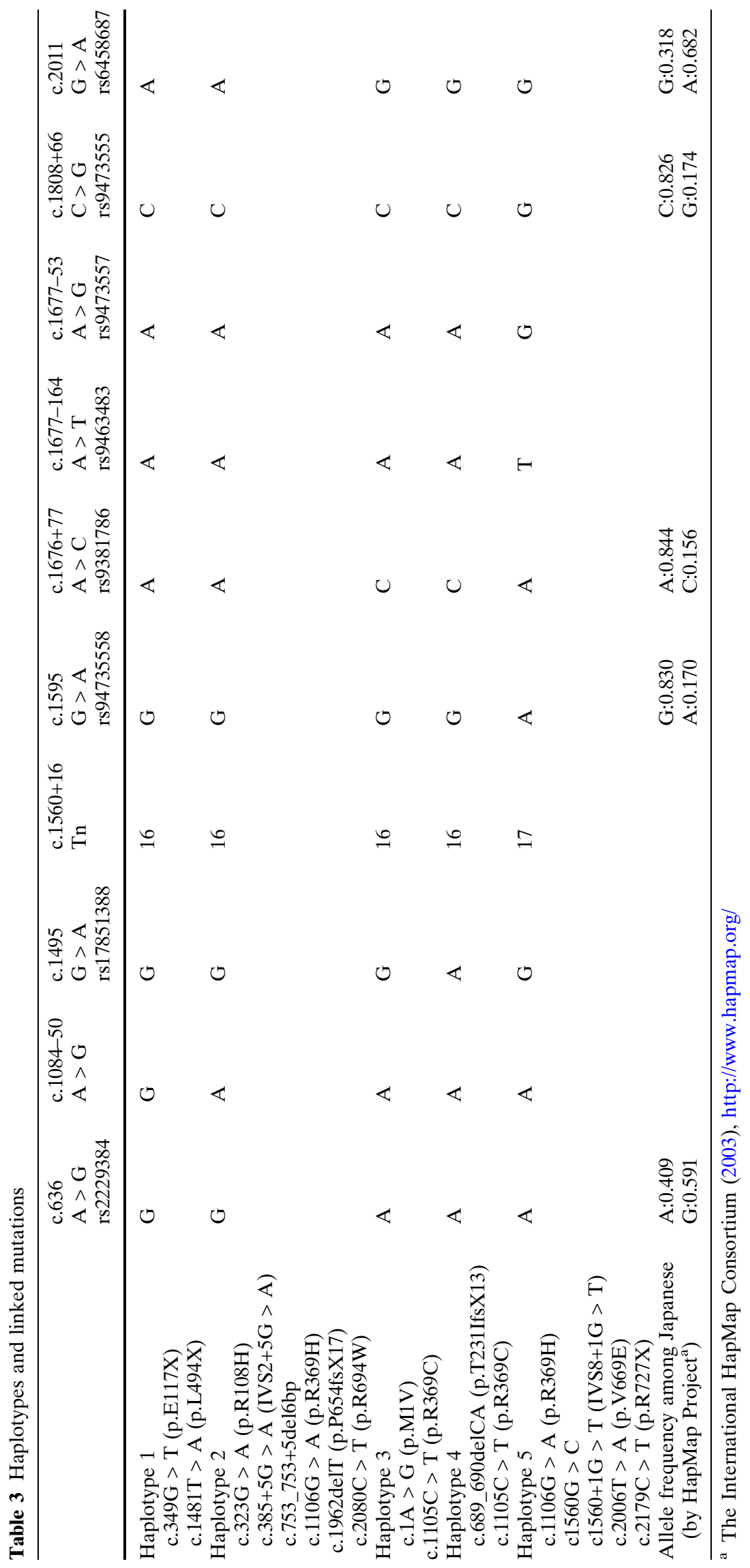

Springer 
With respect to genotype-phenotype correlations, patient 2 was homozygous for the p.E117X and manifested symptoms on the fifth day of life. Ogasawara et al. (1994b) reported a patient homozygous for p.E117X who showed initial symptoms at the age of 9 months. Patients 5 and 6 were homozygous for c. $385+5 \mathrm{G}>\mathrm{A}$, and the onset was neonatal in both cases: patient 5 is still alive (now 7 years old) and patient 6 died after the first attack. Three patients $(7,8$, and 9) were compound heterozygotes of c. $385+5 \mathrm{G}>\mathrm{A}$ and p.R369H: patient 7 manifested symptoms at 8 months and these have been kept under well control with vitamin B12 treatment; patients 8 and 9 both showed initial symptoms in the neonatal period and did not response to vitamin B12. In a previous kinetics study, the $V_{\max }$ value of the p.R369Hmutant enzyme was only $1 \%$ of that of the wild type (Janata et al. 1997), and Toyo-Oka et al. (1995) reported that reverse transcription (RT)-PCR did not demonstrate the presence of a normally spliced transcript from fibroblasts of a homozygote of c. $385+5 \mathrm{G}>\mathrm{A}$. We have no explanation why patient 7 responds to vitamin B12 treatment. The clinical features seem to correlate not only with genotype, but also with other unknown environmental factors, such as the nutritional state and/or modifier genes.

Ten single nucleotide polymorphisms (SNPs) were found in the sequenced region, and the haplotypes could be separated into five groups based on five SNP patterns (Table 3). Among the more frequent mutations, p.E117X and p.L494X were linked to haplotype $1 ;$ c. $385+5 \mathrm{G}>\mathrm{A}$ was linked to haplotype 2 ; p.R727X was linked to haplotype 5 . These frequent mutations appeared to have been spread by the founder effect in the Japanese population. Mutation p.R369H, on the other hand, may be of a double origin (haplotypes 2 and 5). The p.R369 codon contains a CpG dinucleotide, and p.R369H has been found in Turkish, Greek, Caucasian, Hispanic (Worgan et al. 2006), and Korean (Jung et al. 2005) populations.

In conclusion, a limited number of mutations accounted for most of the Japanese mut MMA patients, which is in contrast to the results of a previous study on Caucasian patients. Hopefully the results reported here will facilitate the DNA diagnosis of $m u t$ MMA within the Japanese population.

Acknowledgments We thank all of the physicians who provided samples and/or clinical and biochemical data on their patients. We also express our gratitude to Dr. F. Kok (University of Sao Paulo) for providing us with dried blood samples This work was supported by Grants-in-Aid for Scientific Research from the Ministry of Education, Culture, Sports, Science and Technology (MEXT) of Japan and the Morinaga Hoshikai Foundation.

\section{References}

Acquaviva C, Benoist J-F, Callebaut I, Guffon N, Ogier de Baulny H, Touati G, Aydin A, Porquet D, Elion J (2001) N219Y, a new frequent mutation among mut ${ }^{0}$ forms of methylmalonic acidemia in Caucasian patients. Eur J Hum Genet 9:577-582

Acquaviva C, Benoist J-F, Perwira S, Callebaut I, Koskas T, Porquet D, Elion J (2005) Molecular basis of methylmalonyl CoA mutase apoenzyme defect in 40 European patients affected by mut ${ }^{0}$ and mut $^{-}$forms of methylmalonic acidemia: identification of 29 novel mutations in the MUT gene. Hum Mutat 25:167-176

Adjalla CE, Hosack AR, Matiaszuk NV, Rosenblatt DS (1998) A common mutation among blacks with mut methylmalonic aciduria. Hum Mutat S1:S248-S250

Cheadle JP, Belloni E, Ferrari M, Millar-Jones L, Meredith AL (1994) A novel mutation (M1V) in the translation initiation codon of the cystic fibrosis transmembrane conductance regulator gene, in three $\mathrm{CF}$ chromosomes of Italian origin. Hum Mol Genet 3:1431-1432

Fenton WA, Gravel RA, Rosenblatt DS (2001) Disorders of propionate and methylmalonate metabolism. In: Scriver CR, Beaudet AL, Sly WS, Valle D (eds) The metabolic and molecular bases of inherited disease, 8th edn. McGraw-Hill, New York, pp 2165-2193

Janata J, Kogekar N, Fenton WA (1997) Expression and kinetic characterization of methylmalonyl-CoA mutase from patients with the mut phenotype: evidence for naturally occurring interallelic complementation. Hum Mol Genet 6:1457-1464

Jung JW, Hwang IT, Park JE, Lee EH, Ryu KH, Kim SH, Hwang JS (2005) Mutation analysis of the MCM gene in Korean patients with MMA. Mol Genet Metab 84:367-370

Kakinuma H, Ogura N, Ohtake A, Takayanagi M, Nakajima H, Kondo H, Terada H, Okuda K, Nomoto Y (1985) Biochemical analysis of intact fibroblasts from two cases with methylmalonic acidaemia. J Inherit Metab Dis 8:151-152

Kikuchi M, Hanamizu H, Narisawa K, Tada K (1989) Assay of methylmalonyl CoA mutase with high-performance liquid chromatography. Clin Chim Acta 184:307-313

Kobayashi A, Kakinuma H, Takahashi H (2006) Three novel and six common mutations in 11 patients with methylmalonic acidemia. Pediatr Int 48:1-4

Ledley FD, Rosenblatt DS (1997) Mutations in mut methylmalonic acidemia: clinical and enzymatic correlations. Hum Mutat 9:1-6

Lyonnet S, Melle D, de Braekeleer M, Laframboise R, Rey F, John SW, Berthelon M, Berthelot J, Journel H, Le Marec B (1992) Time and space clusters of the French-Canadian M1V phenylketonuria mutation in France. Am J Hum Genet 51:191-196

Martinez MA, Rincon A, Desviat LR, Merinero B, Ugarte M, Perez B (2005) Genetic analysis of three genes causing isolated methylmalonic acidemia: identification of 21 novel allelic variants. Mol Genet Metab 84:317-325

Mikami H, Ogasawara M, Matsubara Y, Kikuchi M, Miyabayashi S, Kure S, Narisawa K (1999) Molecular analysis of methylmalonyl-CoA mutase deficiency: identification of three missense mutations in mut $^{0}$ patients. $\mathrm{J}$ Hum Genet 44:35-39

Ogasawara M, Matsubara Y, Mikami H, Narisawa K (1994a) Molecular analysis of methylmalonic acidemia: identification of novel mutations in the methylmalonyl-CoA mutase gene with decreased levels of mutant mRNA (abstract). Am J Hum Genet 55:A233 
Ogasawara M, Matsubara Y, Mikami H, Narisawa K (1994b) Identification of two novel mutations in the methylmalonylCoA mutase gene with decreased levels of mutant mRNA in methylmalonic acidemia. Hum Mol Genet 3:867-872

Robinson DO, Bunyan DJ, Gabb HA, Temple IK, Yau SC (1997) A small intraexonic deletion within the dystrophin gene suggests a possible mechanism of mutagenesis. Hum Genet 99:658-662

Rosenblatt DS, Fenton WA (2001) Inherited disorders of folate and cobalamin transport and metabolism. In: Scriver CR, Beaudet AL, Sly WS, Valle D (eds) The metabolic and molecular bases of inherited disease, 8th edn. McGraw-Hill, New York, pp 3897-3933

The International HapMap Consortium (2003) The International HapMap Project. Nature 426:789-796
Toyo-Oka Y, Wada C, Ohnuki Y, Takada F, Ohtani H (1995) Molecular diagnosis of a kindred with novel mutation of methylmalonyl-CoA mutase gene using non-RI SSCP (in Japanese). Rinsho Byori 43:625-629

Worgan LC, Niles K, Tirone JC, Hofmann A, Verner A, Sammak A, Kucic T, Lepage P, Rosenblatt DS (2006) Spectrum of mutations in mut methylmalonic acidemia and identification of a common Hispanic mutation and haplotype. Hum Mutat 27:31-43

Yang X, Sakamoto O, Matsubara Y, Kure S, Suzuki Y, Aoki Y, Suzuki Y, Sakura N, Takayanagi M, Iinuma K, Ohura T (2004) Mutation analysis of the MMAA and MMAB genes in Japanese patients with vitamin B12-responsive methylmalonic acidemia: identification of a prevalent MMAA mutation. Mol Genet Metab 82:329-333 